\title{
Simultaneous surgery is effective treatment and could be the management of choice in patients with infective endocarditis and splenic abscess
}

Hadi abdulsalam Abo Aljadayel ${ }^{1 *}$, Aahmad Kahwaji ${ }^{2}$, Sadegh Zargar Nataj ${ }^{3}$, Amir Jamsid Khamoshi ${ }^{4}$ and Kourosh Trigarfakheri $^{5}$

${ }^{1}$ Master cardiac surgery, Department of Syrian and Arab board cardiac surgery, Syria

${ }^{2}$ Syrian board in general surgery, Syria

${ }^{3}$ Iranian board in cardiac surgery, Iran

${ }^{4}$ Iranian board in cardiac surgery, Iran

${ }^{5}$ Iranian board of anesthesia, Iran

\begin{abstract}
The treatment of patients with IE and splenic abscesses is still challenging and controversial. We present tow case reports about our management of patients diagnosed with infective endocarditis and have splenic abscesses. Our experience confirms that in such a patient, heart operation combined with splenectomy can be performed simultaneously with excellent results, lifesaving, and free of complications during the same anesthesia and could be the management of choice.
\end{abstract}

\section{Introduction}

There are several reports that $3-5 \%$ of all patients with infective endocarditis (IE) are complicated by the development of splenic abscess, which is a rare but well-known adverse event [1-3].

\section{Case report 1}

A 60-years-old male, who transferred to our center emergently with diagnosis of endocarditis complaining mainly from fever and general fatigue was admitted for evaluation in another hospital for 10 days before because of his low-grade fever and general fatigue and in which he took medical therapy and then transferred to our center because of deterioration in his general situation. After accepting in CCUEmergency and volume resuscitation a head, thoracic and abdominal computed tomography (CT) scan was done which revealed splenic abnormality (Figure $1 \mathrm{~A} 1$ ). Transthoracic echocardiography revealed many low echoic hypermobile lesions (vegetation's) on Aortic, Mitral, Tricuspid valve leaflets with severe regurgitation for all tree valves (Figure 1 A6), left ventricular systolic function was normal. CT scan of the brain was normal. physical examination was as follows: blood pressure, $80 / 30 \mathrm{mmHg}$, heart rate, $135 \mathrm{~b} / \mathrm{m}$; body temperature, $38.3^{\circ} \mathrm{C}$; $\mathrm{SpO}_{2}, 91 \%$ (room air). Blood examination values $\mathrm{WBC} 15,600 \mathrm{c} / \mathrm{l}, \mathrm{hb}$ $9.2 \mathrm{~g} / \mathrm{dl}$, plt 189,000 cells/l, CRP concentration $11.5 \mathrm{mg} / \mathrm{dl}$. In addition, blood culture from another hospital was positive for Enterococcus faecalis for which the patient was taking antibiotic treatment for 10 days with sulbactam/ampicillin (SBT/ABPC) and gentamycin.

\section{Case report 2}

A 39-year-old male patient was transferred to our center with diagnosis of infective endocarditis and splenic abscesses for further management. The patient presented initially with prolonged fever, shortness of breath, fatigue. His initial echocardiography revealed severe aortic regurgitation and vegetation attached to the ventricular surface of aortic valve leaflets (Figure 2 B2). His blood culture was positive for enterococcus faecalis. Physical examination: blood pressure, $95 / 45 \mathrm{mmHg}$, heart rate, $100 \mathrm{~b} / \mathrm{m}$; body temperature, $38.3^{\circ} \mathrm{C}$; $\mathrm{SpO}_{2}, 94 \%$ (room air). WBC15,600 c/l, Hb $8.6 \mathrm{~g} / \mathrm{dl}$, plt 219,000 cells/l, CRP concentration $13.5 \mathrm{mg} / \mathrm{dl}$. High Pro brain natriuretic peptides (Pro-BNP) indicating significant heart failure. Computed tomography (CT) scan of the abdomen, brain, confirmed the presence of splenic lesion which could most likely be abscess (Figure 2 B1).

\section{Operation technique for both cases}

After evaluation and discussion by a multidisciplinary team comprising cardiac surgeons, cardiologists, general surgeons, and infectious disease specialists, the decision for a splenectomy and heart surgery was carried out to do simultaneously at the same anesthesia. After complete splenectomy by midline incision we packed the spleen bed with long gauze and approximate the skin with some stitches then were re-sterile the patient for routine cardiac surgery.

Case 1: Because of emergent situation we accepted to operate without evaluate the coronary arteries. Because of Aortic valve vegetations we could not achieve angiography, and because of tachycardia which is not controllable by the exists of severe AI and high levels of Pro brain natriuretic peptides (Pro-BNP) which refer to

*Correspondence to: Hadi abdulsalam Abo Aljadayel, Master cardiac surgery, Department of Syrian and Arab board cardiac surgery, Syria, E-mail: hadyaboaljadayel@yahoo.com

Key words: infective endocarditis, splenic abscess, simultaneous operation

Received: January 09, 2020; Accepted: January 20, 2020; Published: January 27, 2020 


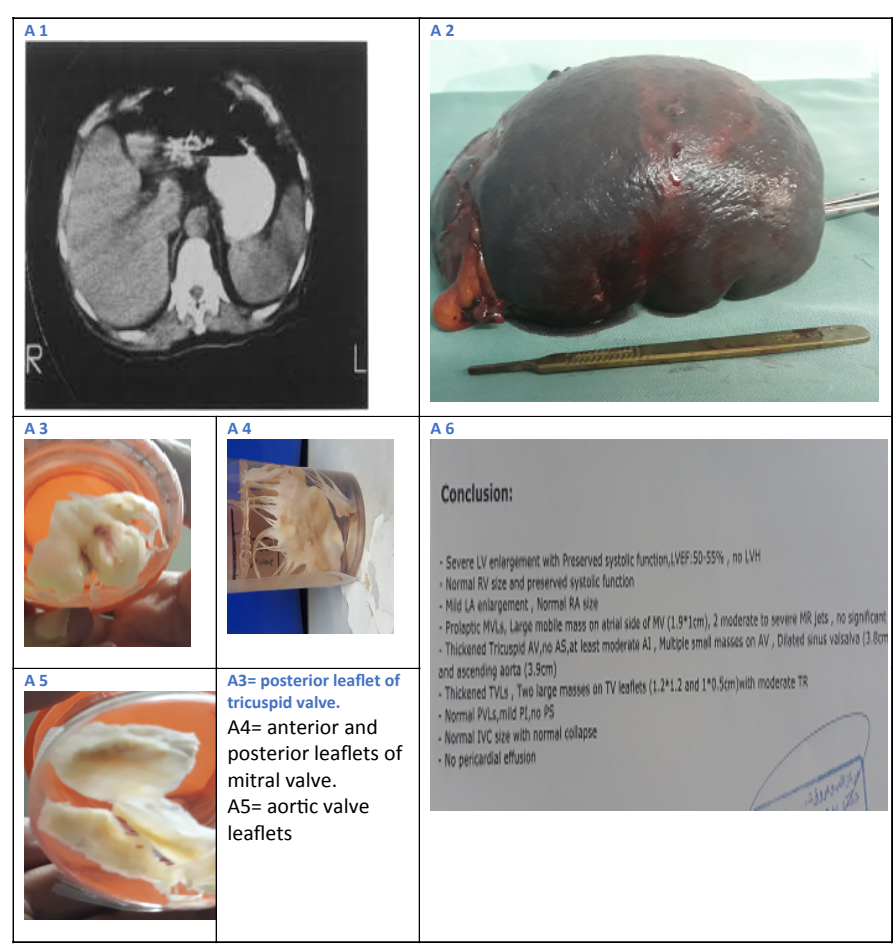

Figure 1. Case 1, CT abdomen reveal the splenic abscess A1, the spleen after operation A2, the vegetation's on the valves leafletsA3,4,5 and the conclusion of echocardiography before operation.

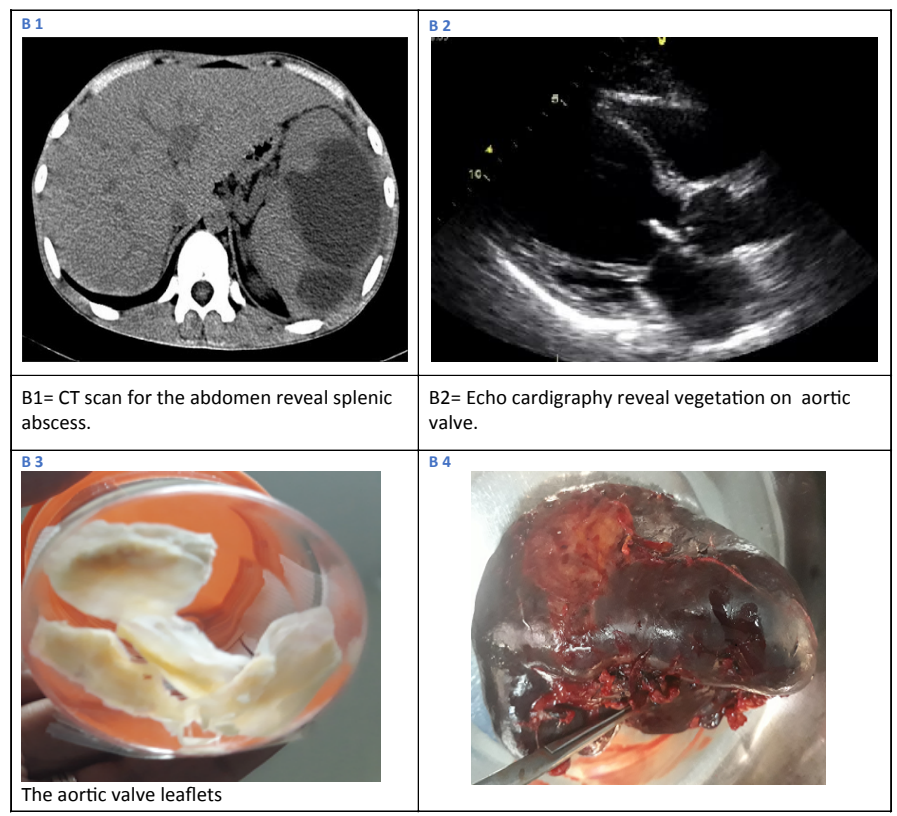

Figure 2. B1, CT scan of abdomen reveal splenic abscess. B2, echocardiography for aortic valve see the vegetation's. B3, the aortic valve leaflets as specimens. B4 the spleen.

heart failure we couldn't do CT angio for the patient. We replaced the aortic valve with bioprosthetic valve 23 and the mitral valve with 31 bioprosthetic, there was vegetation's on the posterior leaflet only, so we excised this leaflet and plicate the annulus (bicusbidisation). With my finger I checked the whole tract of coronary arteries which revealed calcified plaque on proximal LAD caused severe stenosis in the artery when checked by probe, so we put vain graft bypass Aorta to LAD after that plaque.
Case 2: Underwent successful aortic valve replacement by metallic valve 23 , followed by an uneventful postoperative course.

\section{Discussion}

The order of splenectomy and valve replacement might influence the outcome. As most previous articles believe that more data are required to state the recommended order of operations [1-8] our case reports add additional evidence for successfully treating IE complicated with SA. Patients with IE complicated with SA and showed that medical treatment alone resulted in a poor outcome, while treatment with splenectomy resulted in a high survival rate $[1,4]$. Splenectomy is thought to be essential for eliminating the potential for prosthetic valve infection after valve replacement. There are three ways to perform the operations: to perform simultaneously in which physical stress might be considerable. To perform the splenectomy followed by valvular surgery its problems may be encountered with the development of an immune compromised condition and a tendency for bleeding. If valvular surgery has done first, there is possibility of prosthetic valve infections because of the existence of SA $[2,4,6]$. Successful one-stage procedures (splenectomy combined with valve replacement) have also been described in the literature and also had good results [5,6]. But there is still thinking that splenectomy should done few days before valve surgery which we want to prove that one step simultaneous surgery is safe and curable and could be management of choice. In old patients it is very important to evaluate coronary arteries but sometimes it's difficult to do like in our unstable patient and because of Aortic valve vegetation we couldn't do angiography also as the patient had severe AI, heart failure and uncontrolled tachycardia we couldn't achieve MCT angiography.

Adequate antibiotic treatment should accompany surgery Antimicrobial regimens should therefore be based on individual blood cultures and perioperative cultures [4]. We sent the spleen specimen and the removed valves leaflets (Figure $1 \mathrm{~A} 2,3,4,5$ ) for pathology and for microbial culture and sensitivity and we modified the antibiotic treatments up to the results when needed (Figure 2 B3,4). The spleen may be affected either by bacteremia seeding an infarcted splenic zone or directly through seeding of the spleen by infected embolized vegetations of the heart valves [5]. Typical symptoms of SA are reported to be fever and abdominal pain and clinical findings are splenomegaly, leukocytosis, and left pleural effusion [3-5]. However, most cases present with a wide range of clinical manifestations, presumption of SA is essential to make the diagnosis in a patient with IE $[3,5]$. Abdominal CT scan and MRI are considered gold standard techniques for diagnosis of splenic abscess, with sensitivity and specificity varying between $90 \%$ and $95 \%[2,6]$ in our patients we did CT scan. We think that more data are required to state the recommended order of operations and could be management of choice. Our case report is clinically important as a part of evidence for successfully treating IE complicated with SA.

\section{Conclusion}

Splenectomy and valve replacement have become standard strategies as medical treatments give unsatisfactory results. However, it has not been established which of these procedures should be carried out first but, in such patients, we believe that simultaneous procedure is the effective, safe and free of complications and could be management of choice.

\section{Disclosures}

The authors have no conflicts of interest to disclose. 


\section{References}

1. Baddour LM, Wilson WR, Bayer AS, Fowler VG Jr, Bolger AF, et al. (2005) Infective endocarditis: diagnosis, antimicrobial therapy, and management of complications: a statement for healthcare professionals from the Committee on Rheumatic Fever, Endocarditis, and Kawasaki Disease, Council on Cardiovascular Disease in the Young, and the Councils on Clinical Cardiology, Stroke, and Cardiovascular Surgery and Anesthesia, American Heart Association: endorsed by the Infectious Diseases Society of America. Circulation 111: e394-434. [Crossref]

2. Seeger M, Folsch UR (2004) Diagnosis of acute splenic infarction by echosignal enhanced ultrasound. Dtsch Med Wochenschr 129: 876-879. [Crossref]

3. Elasfar A, AlBaradai A, AlHarfi Z, Alassal M, Ghoneim A, et al. (2015) Splenic abscess associated with infective endocarditis; Case series. J Saudi Heart Assoc 27: 210-215. [Crossref]
4. Yoshikai M, Kamachi M, Kobayashi K, Murayama J, Kamohara K, et al. (2002) Splenic abscess associated with active infective endocarditis. Jpn J Thorac Cardiovasc Surg 50: 478-480. [Crossref]

5. Ebels J, Van Elst F, Vanderveken M, Van Cauwelaert R, Brands C, et al. (2007) Splenic abscess complicating infective endocarditis: three case reports. Acta Chir Belg 107: 720-723. [Crossref]

6. Simsir SA, Cheeseman SH, Lancey RA, Vander Salm TJ, Gammie JS (2003) Staged laparoscopic splenectomy and valve replacement in splenic abscess and infective endocarditis. Ann Thorac Surg 75: 1635-1637. [Crossref]

7. Saxena P, Boyt A, Newman MA (2009) Mitral Valve Leaflet Abscess Complicating Infective Endocarditis. Heart Lung Circ 18: 133-162. [Crossref]

8. Naito R, Mitani H, Ishiwata S, Yamaguchi T, Tanaka K, et al. (2010) Infective endocarditis complicated with splenic abscess successfully treated with splenectomy followed by double valve replacement. $J$ Cardiol Cases 2: e20-e22. [Crossref]

Copyright: ${ }^{0} 2020$ Aljadayel HAA. This is an open-access article distributed under the terms of the Creative Commons Attribution License, which permits unrestricted use, distribution, and reproduction in any medium, provided the original author and source are credited. 